\title{
LEUCOGRAMA, ERITOGRAMA, HEMOGLOBINA, HEMATÓCRITO E TEMPO DE COAGULAC̣ĀO APÓS O USO ORAL E INTRAPERITONEAL DE Heterothalamus brunioides, EM RATOS WISTAR.
}

Ana Maria Chagas e cloé Mendonça Saldanha

Núcleo de Pesquisa de Produtos Naturais. Centro de Ciências Naturais e Exatas. UFSM. Santa Maria, RS.

Carlos Mario Cunha

Departamento de Análises Clīnicas do Hospital Veterinärio. Centro de Ciências Rurais. UFSM. Santa Maria, RS.

Heleonora B. Vieira, Janice Sitya e Mario Kurtz Filho

Bolsistas Colaboradores e de Iniciação à Pesquisa. UFSM. Santa Maria, RS.

RESUMO

No presente trabalho pretendeu-se observar as possíveis alterações sanguỉneas produzidas pela administração via oral ( $98 \mathrm{mg} /$ $\mathrm{kg}$ ) e via intraperitonial (40 mg/kg e $98 \mathrm{mg} / \mathrm{kg}$ ) de Heterothazamus brunioides Less, durante intervalos de tempo, em ratos Wistar. A anālise dos resultados revela que não houve alterações significati vas nos parāmetros estudados.

\section{SUMMARY}

CHAGAS, A.M.; SALDANHA, C.M.; CUNHA, C.M. ; VIEIRA, H.B. SITYA, J.and KURTZ FILHO, M. 1987. Leukocyte, Erythrocyte, Hemoglobin Concentration, Hemotocrit and Bleeding Cotting Counter by Prolonged oral and intraperitonial used of Heterothazamus brunioides in Wistar rats. Ciência e Natura, 9:67-75,1987.

In the present work, authors wished to observe possible blood alterations produced by oral route (98 $\mathrm{mg} / \mathrm{kg}$ weight) and intra peritonial route $(40 \mathrm{mg} / \mathrm{kg}$ and $98 / \mathrm{kg}$ weight) prolonged administrations of HeterothaZamus brunioides, Less in Wistar rats. Results analysis proved that, in the estudied paramethers, was no significant alte rations.

INTRODUÇÃO

Atualmente os acidentes por picada de serpentes são ainda um problema médico de considerāvel magnitude. Em 1975 SWAROOP e COLS (21), estimaram que 30.000 a 40.000 pessoas morriam anualmente, viti mas de envenenamento por serpentes peçonhentas.

Existem 2.500 espécies de serpentes no mundo, mas menos de 200 delas são venenosas para o homem (15).

Os venenos de serpentes são os mais complexos de todos os venenos conhecidos. Devido as diversas atividades biológicas de seus vārios componentes, os venenos ofídicos produzem efeitos tóxicos e 
letais no sangue e sistemas cardiovascular, nervoso e respiratörio (14).

O Heterothalamus brunioides Less, vulgarmente denominado de "Alecrim do campo", que é entretanto, a nossa melhor "arnica da serra", usada como sucedāneo da "arnica da Europa", è um pequeno ar busto cespitoso, com caules lenhosos, despido na metade inferior e bastante ramificado e ornado de folhas estreitas e lineares na par te superior. Estas, ao serem trituradas depois de secas despreendem uın cheiro agradāvel. (9), Figura 1.

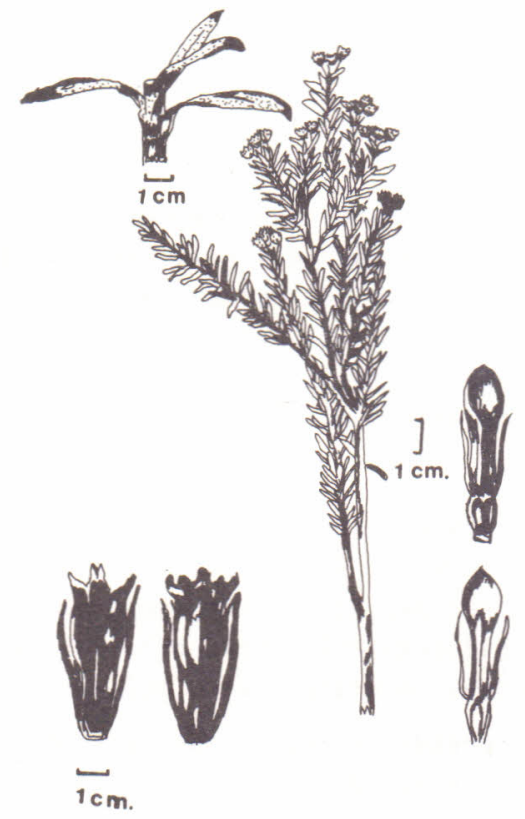

Figura 1 - HeterothaZamus brunioides Less. "Alecrim do Campo" ou "Arnica da Serra".

o Heterothalamus brunioides Less è um arbusto nativo do Rio Grande do Sul; é utilizado popularmente na região sul como con tra-veneno nos empeçonhamentos produzidos pelas serpentes Bothrops. Veneno este considerado por alguns autores (3, 13) como tendo "in vitro" ação anticoagulante.

Segundo ROSENFELD (17), o tipo de ação fisiopatolōgica do envenenamento pelo gênero Bothrops ocorre por uma ação proteolỉtica e uma ação coagulante. Esta ocorre pela coagulação do fibrinogênio que se deposita em microcoāgulos, que se chocam entre si, e poste riormente se depositam a nỉvel dos capilares pulmonares. Nesta fase, o sangue fica friāvel ou seja incoagulāvel devido a falta de fibri nogênio (7). A ação proteolîtica ocorre por ação das hemorraginas 
(proteolisinas), que agem causando inicialmente vasoconstrição arte riolar e posteriormente vasodilatação e hemorragia do leito capilar. Devido a esta vasodilatação os eritrocitos escapam entre as células endoteliais, logo a membrana basal fica rompida e permite o extrava samento de 1 îquidos (14).

Pensando-se na possibilidade do Heterothalamus Brunioides apresentar um efeito benéfico, como cita a Medicina popular, contra o veneno de serpentes, e como observamos acima ser o sistema sangu $\underline{i}$ neo o mais envolvido no quadro tóxico, os autores objetivam avaliar se o extrato aquoso do referido vegetal produz algum efeito no le cogrania, eritograma, hematōcrito, hemoglobina e no tempo de coagula ção após o uso prolongado pela via oral ou intraperitonial.

MATERIAL E MÉTODO

Preparo do Extrato: O Heterothalamus brunioides, foi cole tado no municipio de Santaninha da Boa Vista e identificado segundo HOEHNE (9). Após coletado e seco ao ar, foi selecionado e triturado em liquidificador, o extrato obtido foi mantido a $4^{\circ} \mathrm{C}$, como solução mãe.

Procedimento: As experiências foram realizadas em 180 ra tos Wistar machos, com 90 dias de idade e peso médio de 165 gramas, mantidos no Biotério do Departamento de Fisiologia a uma temperatu ra de $24^{\circ} \mathrm{C} \pm 2$, com āgua e alimento a vontade. Os animais foram di vididos em 15 grupos, conforme o protocolo experimental. Figura 2.

Apesar de CRESKOFF e COLS (5), não encontrarem diferenças significativas na contagem de leucōcitos entre os sexos, utilizamos somente ratos machos. Foram utilizados animais de mesma raça e ida de (TABELA I, II e III) uma vez que RINGLER e DABICH (16) comprova ram existir alteraçöes significativas no total de leucōcitos em re lação a idade e raça.

Os experimentos foram divididos em duas etapas, sendo que na primeira utilizou-se a via oral e na segunda a via intraperito nial para a administração do extrato de Heterothazamus brunioides.

os grupos 1, 6 e 11 (com animais cada grupo) foram os con troles e receberam somente àgua e alimento a vontade; deste, reti rou-se amostras sanguineas nos dias 0 .

Os 40 animais que totalizaram os grupos $2,3,4$ e 5 além de alimento receberam $98 \mathrm{mg} / \mathrm{kg}$ je extrato aquoso de Heterothazamus brunioides pela via oral e o ingeriam a vontade por 30,60, 90 e 120 dias respectivamente e, nestes dias foram coletadas amostras sangu neas.

Os 40 animais que totalizavam os grupos $7,8,9$ e 10 rece beram āgua e alimento a vontade e administração diāria de Heterotha Zamus brunioides na concentração de $40 \mathrm{mg} / \mathrm{kg}$ pelä via intraperitonial 
durante $5,10,20$ e 40 dias respectivamente e as coletas sanguineas foram efetuadas nos dias $0,5,10,20$ e 40.

I ETAPA (Vio oral)

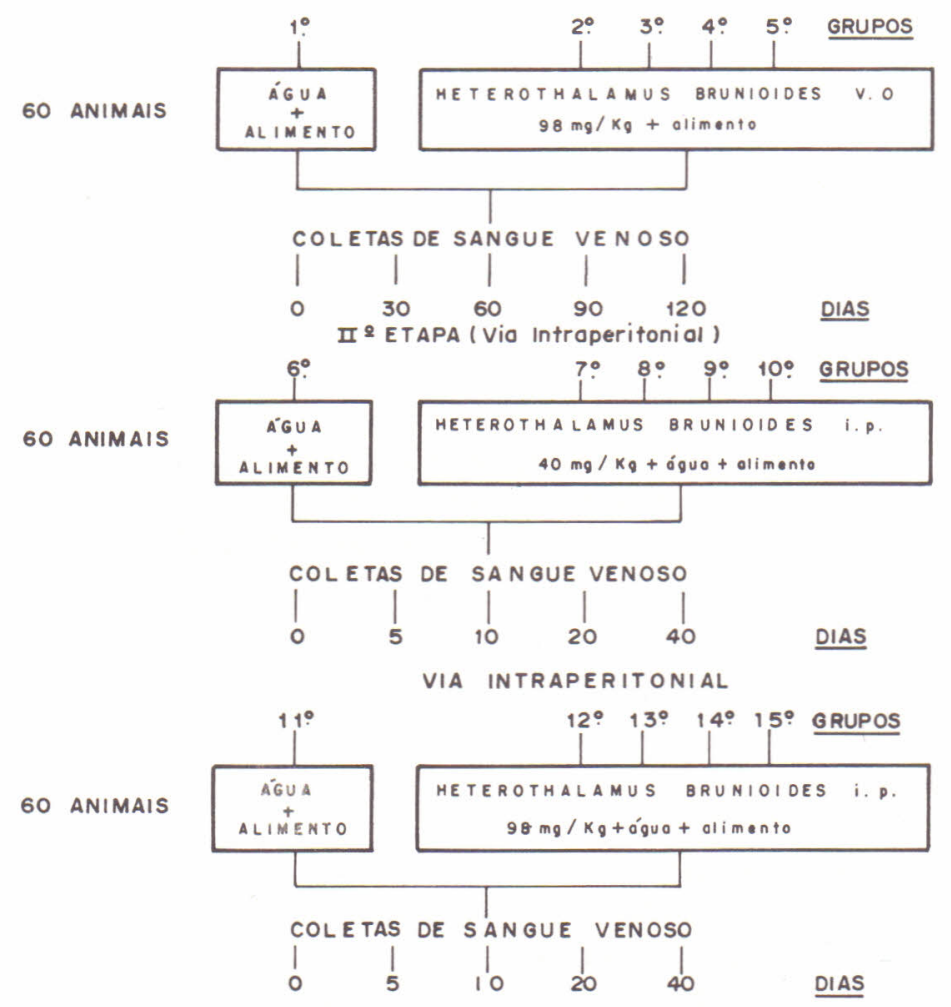

Figura 2 - Protocolo Experimental.

Em todos os grupos utilizamos para coletar o sangue a veia da cauda ou do plexo venoso no āngulo interno do olho (8) e para is to os animais foram anestesiados com pentobarbital sódico (45 mg/ $\mathrm{kg})$.

As contagens de eritrōcitos, hematōcrito, hemoglobina, leu cōcitos foram efetuados através do aparelho Coulter Counter Eletro nics Ind Comer. Ltda, no mesmo dia da coleta, da mesma maneira que o realizado por CHAGAS e COLS (4). O tempo de coagulação foi efetua do segundo a técnica de MILIAN-GREENBERG, (especificações de FISHER 6)

Para avaliar as alterações produzidas nos diversos casos utilizou-se o teste "T" de Student (SNEDECOR e COCHRAN,20). 
Heterothalamus brunioides na concentração utilizada, pela via oral em diferentes tempos não produz alterações significativas no leuco grama, eritograma, hemoglobina, hematōcrito e no tempo de coagulação.

TABELA I - VALORES MEDIOS DO LEUCOGRAMA EM $\mathrm{mm}^{3}$, ERITOGRAMA EM MILHOES/m ${ }^{3}$. DA HEMOGLOBINA EM GRAMAS/OI DO HEMA TOCRITO EM PERCENTUAL E DO TEMPO DE COAGULAÇAO EM SEGUNDOS DO SANGUE VENOSO DE RATOS ANTES E APOS A ADMINISTRAÇAO VIA ORAL DE Heterothatamus brunioides $98 \mathrm{mg} / \mathrm{kg}$ DURANTE $30,60,90$ E 120 DIAS.

\begin{tabular}{|c|c|c|c|c|c|c|c|c|c|c|c|c|c|c|c|}
\hline \multirow{2}{*}{$\begin{array}{l}\text { Parâmetros } \\
\text { Leocograma }\end{array}$} & \multicolumn{3}{|c|}{$\begin{array}{l}\text { àgua }+ \text { alimento } \\
\text { controle }\end{array}$} & \multicolumn{6}{|c|}{$\begin{array}{l}\text { E.A.Hb + alimento } \\
30\end{array}$} & \multicolumn{3}{|c|}{90} & \multicolumn{3}{|c|}{120} \\
\hline & 12460 & \pm 320 & $(20)$ & 12140 & \pm 380 & $(40)$ & 14470 & \pm 520 & $(40)$ & 12640 & \pm 180 & $(40)$ & 15280 & \pm 790 & $(40)$ \\
\hline Eritograma & 7,58 & $\pm 1,2$ & $(20)$ & 7,58 & $\pm 1,6$ & $(40)$ & 8,37 & $\pm 1,5$ & $(40)$ & 7,62 & $\pm 2,4$ & $(40)$ & 8,00 & $\pm 1,9$ & $(40)$ \\
\hline Hemoglobina & 16,9 & $\pm 2,9$ & $(20)$ & 15,9 & \pm 2.9 & $(40)$ & 17.2 & $\pm 2,1$ & $(40)$ & 17,6 & $\pm 1,8$ & $(40)$ & 17,8 & $\pm 1,2$ & $(40)$ \\
\hline Hema tócrito & 40,0 & $\pm 1,2$ & $(20)$ & 43,2 & $\pm 2,7$ & $(40)$ & 41.8 & $\pm 2,4$ & $(40)$ & 42,6 & $\pm 1,5$ & $(40)$ & 41,8 & $\pm 1,7$ & $(40)$ \\
\hline T. Coagulação & 58,0 & $\pm 2,0$ & $(20)$ & 65,0 & $\pm 5,0$ & $(40)$ & 60,0 & $\pm 7,0$ & $(40)$ & 58.0 & \pm 6.0 & $(40)$ & 64,0 & $\pm 1,7$ & $(40)$ \\
\hline
\end{tabular}

E. A.Hb = Extrato aquoso de Heterothalamus brunioides.

$($ ) = Nümero de animais.

TABELA II - VALORES MEDIOS DO LEUCOGRAMA EM $\mathrm{mm}^{3}$, ERITOGRAMA EM MILHOES/mm ${ }^{3}$, DA HEMOGLOBINA EM GRAMAS/dI DO HE MATOCRITO EM PERCENTUAL E DO TEMPO DE COAGULAÇAO EM SEGUNDOS DO SANGUE VENOSO DE RATOS ANTES E APOS A ADMINISTRAÇAO VIA INTRAPERITONIAL DE Heterothalamus brunioides $98 \mathrm{mg} / \mathrm{kg}$ DURANTE $5,10,20$ E 40 DIAS.

\begin{tabular}{lccccc}
\hline Parâmetros & $\begin{array}{l}\text { ăgua }+ \text { alimento } \\
\text { controle }\end{array}$ & 5 & 10 & 20 & 40 \\
\hline Leucograma $(40)$ & $12460 \pm 320$ & $14890 \pm 530$ & $11760 \pm 530$ & $12680 \pm 410$ & $15278 \pm 720$ \\
Eritograma (40) & $7,58 \pm 1,20$ & $7,90 \pm 2,20$ & $7,00 \pm 2,47$ & $8,40 \pm 2,60$ & $7,32 \pm 1,80$ \\
Hemoglobina (40) & $16,8 \pm 1,80$ & $15,8 \pm 2,90$ & $17,3 \pm 2,70$ & $16,6 \pm 2,50$ & $17,2 \pm 3,20$ \\
Hematöcrito (40) & $40,0 \pm 1,20$ & $41,9 \pm 2,10$ & $49,9 \pm 1,20$ & $42,8 \pm 2,10$ & $43,8 \pm 2,70$ \\
T. Coagulação (40) & $58,0 \pm 2,0$ & $52,0 \pm 4,0$ & $55,0 \pm 2,0$ & $64,0 \pm 6,0$ & $57,0 \pm 3,0$ \\
\hline
\end{tabular}

E.A.HB = Extrato aquoso de Heterothalamus brunioides.

$($ ) = Nümero de animais.

o Heterothalamus brunioides quando administrado pela via 
não apresenta alterações significativas em nenhum dos parâmetros es tudados.

TABELA III - VALORES MEDIOS DO LEUCOGRAMA EM $\mathrm{mm}^{3}$. ERITOGRAMA EM MILHOES/mm ${ }^{3}$. DA HEMOGLOBINA EM GRAMAS/dI DO HE MATOCRITO EM PERCENTUAL DO TEMPO DE COAGULAÇAO EM SEGUNDOS DO SANGUE VENOSO DE RATOS ANTES E APOS A ADMINISTRAÇAO INTRAPERITONIAL DIARIA DE Heritothalamus brunioides $40 \mathrm{mg} / \mathrm{kg}$ DURANTE $5,10,20$ E 40 DIAS.

\begin{tabular}{|c|c|c|c|c|c|c|c|c|c|}
\hline \multirow{2}{*}{ Parämetros } & \multirow{2}{*}{$\begin{array}{l}\text { água }+ \text { alimento } \\
\text { controle }\end{array}$} & & \multicolumn{3}{|c|}{ E.A. Hb + alimento } & \multirow{2}{*}{\multicolumn{2}{|c|}{20}} & \multirow{2}{*}{\multicolumn{2}{|c|}{40}} \\
\hline & & & 5 & & 10 & & & & \\
\hline Leucograma (40) & $12460 \pm 320$ & 14970 & \pm 660 & 13870 & \pm 320 & 12270 & \pm 490 & 13670 & \pm 280 \\
\hline Eritrócitos $(40)$ & $7,58 \pm 1,20$ & 7,00 & $\pm 2,80$ & 8,37 & $\pm 2,80$ & 7,50 & $\pm 2,90$ & 7,07 & $\pm 2,60$ \\
\hline Hemoglobina $(40)$ & $16,8 \pm 1,80$ & 17,1 & $\pm 3,20$ & 16,0 & $\pm 2,10$ & 16,5 & $\pm 3,70$ & 16,9 & $\pm 1,20$ \\
\hline Hema töcrito $(40)$ & $40,0 \pm 1,20$ & 42,7 & $\pm 2,20$ & 43,8 & \pm 1.80 & 44,0 & $\pm 2,80$ & 41,8 & $\pm 1,70$ \\
\hline T. Coagulação (40) & $67,0 \pm 3,0$ & 62,0 & $\pm 4,0$ & 58,0 & $\pm 5,0$ & 64,0 & $\pm 7,0$ & 60,0 & $\pm 9,0$ \\
\hline
\end{tabular}

E. A. Hb = Extrato aquoso de Heterothalamus brunioides,

( ) = Nümero de animais.

\section{DISCUSSAO E CONCLUSOES}

Comparando as alterações dos leucócitos nos animais que receberam o Heterothalamus brunioides pela via oral ou intraperito nial com os controles que receberam somente āgua e alimento, obser vou-se não serem as mesmas significativas, pois as variações existen tes permaneceram entre valores fisiológicos aceitos por SCHLAM e COLS (18).

O eritograma em todos os animais não apresentou alterações significativas, isto mostra que o Heterothalamus brunioides não al tera a sērie vermelha quando administrado pela via oral ou intrape ritonial. As variações existentes permanecem dentro dos parāmetros fisiológicos encontrados por outros autores (5, 11, 12, 23). Tabela I, II e III.

A concentração da hemoglobina dos animais que receberam o Heterothalamus brunioides pela via oral ou intraperitonial em compa ração com os controles não mostraram alterações significativas. Se gundo SCHERMAN (19) a concentração de hemoglobina em ratos pode va riar de 11,4 a $19,2 \mathrm{~g} / \mathrm{d} 1$ dados estes confirmados por outros autores $(2,8,19,22,24)$ e alterações semelhantes ocorreram em nossos achados, conforme Tabelas I, II e III.

o hematócrito tambēm não apresentou alterações significa tivas com os tratamentos efetuados e assim, como no homem o hematöcrito 
é aproximadamente trés vezes o valor da hemoglobina que varia de $40,5 \%$ a $53,9 \%$ (16), Tabelas I, II e III.

0 tempo de coagulação foi efetuado pelo teste de MILIANGREENBERG citado por FISHER (6), tomando todos os cuidados especif $i$ Cados por AZZOLIN e COLS (1); os animais tratados não apresentaram al terações em relação ao controle. De acordo com CRESKOFF e COLS (5) o tempo de coagulação é de aproximadamente 2 minutos. Tabelas I, II e II I.

Analisando todos os resultados obtidos no presente traba lho, pode-se concluir que não houve alteração significativa para ne nhum parâmetro proposto.

Talvez o efeito benéfico ocorra nos animais intoxicados, mas em animais normais os parâmetros sanguỉneo não se alteram. A realização deste trabalho se prende ao fato de que a administração consecutiva e diāria poderia alterar outros parāmetros e causar pro blemas posteriores.

\section{AGRADEC IMENTOS}

A Profa. Maria Lacy Weis, do Departamento de Biologia da UFSM, pela revisão completa do texto, sugestões e modificações apre sentadas.

\section{BIBL IOGRAF IA C ITADA}

1. AZZOL In, E.L.C., SimOES, S.R.M. and ChAGAS, A.M. - Métodos de Ava liação do Tempo de Coagulação Sanguỉnea em Ratos Wistar. 2 a Jornada de Cirnica Médica de Santa Maria, RS, 1986.

2. BAUMGARTNER, R. and LABER, G. - Haematologische untersuchunger biratten mit experimentelier myhoplasma polyarthritis. Zen tralberl Babesial parastenkd infektionshr. Hyg Abt I Reihe A, 232: $105-12,1975$.

3. BRASIL, O.V. FRANCESCHI, J.P. and WAISBICH, E. - Pharmacology of crystalline crotoxim I. toxicity. Men Inst Butantan Simp Intermac, 33:973-980, 1966.

4. ChAGAS, A.M. SALDANHA, C. and OLIVEIRA, P.R. - Leucometria, Eri trometria e os Teores Plasmáticos de Sódio, de Potássio e de Cálcio, Apōs o uso prolongado de Ramaria flavo bmunescens em Ratos. Rev. Centro Ciências Rurais, 10(4), 299-306, 1980.

5. CRESKOFF, A.J., FRITZ-HUGH, T. JR and FARRIS, E.J. - Hematology of the Rat and Standards in "The Rat in Zaboratory investiga tion" (E.J. FARRIS and J.Q. GRIFFITHS, eds) pp. 406-420, $1 \underline{i}$ pincott. Philadelphia, Pensylvania, 1949.

6. FISHER, A. - Laboratório de Anälises Clinicas, 6ă ed, Buenos Ai res, Editora El Ateneo, 1954, $246 \mathrm{p}$.

7. FONTANA, F. - Traité sur le Vénin de la Vipere sur les Poisons Americains sur le Lausier Gerise et sur quelques outres Poisons 
Vegetalaux. Vol I and III Firinze, 1781.

8. HEBOLD, V.G. and BLEUEL, H. - Hämatologische standard werte bei der weiblichen und mamlichen ratte (Sprague Dawley). 2 . Ver suchstierkd, 13: 316-320, 1971.

9. HOEHNE, F.C. - Plantas e substâneias vegetais töxicas e mediei nais. São Paulo, graphcars, 1939, 352 p.

10. HULSE, E.V. - Quantitative cell counts of the bone marrow and blood their secular variations in the normal adult rat. Acta Haematol. $31: 50-63,1964$.

11. JONES, R.A., JENKINS, L.J. JR., COON, R.A. and SIEGEL, J. - Effect of long-term continuous inhalation of ozone on experimental animals. Toxicol appe Pharmacol, 17: 189-202, 1970.

12. KOSMA, C.K., WEISBROTH, S.H., STRATMAN, S.L. and CONEJEROS, M. Normal Biologic valus for long. Evans rats Lab anim. Care, 19: $746-55,1969$.

13. LACERDA, F. - Are Museu Nac. Rio de Janeiro. Ed. Guanabara, 1978, $202 \mathrm{p}$.

14. OHSAKA, A. - Hemorragic Necrotizing and Edema: Forming effects of Snake Venouns. Chapter 14, Washington Mac Graw Hill, 1975 , $503 \mathrm{p}$.

15. POLLARD, C.B. - Venon Research Ghalienge to Various Sciences in Venouns. ed. by E.E. Buchey and N, porges publication 44. Am Assoc. Advancement of Science, Washington, D.C. 1956, 5-8 p.

16. RINGLER, D.H. and DABICH, L. - Hematologic and clinical, Bioche mistray. The Laboratory Rat, 1:105-121, 1979.

17. ROSENFELD, G. - Symptomalogy, Pathology and Tratament of snake bites in South Amērica. In venouns animals and their venouns. Vol II, New York academic pres. 1971, Biochemistray. The Labo ratory Rat, 105-121, 1979.

18. SCHALM, O.W., JAIN, N.C. and CARROLL, E.J. - Veterinary Hematozo gy. 3rd ed Lia and Finger, Philadelfia, Pensylvania, 1975, 278p.

19. SCHERMAN, H. - Comparative Profilis of Various Strains of Rats Used in long-term juding Studies. Lab anim. Care, 13:793-807, 1963.

20. SNEDECOR, G.W. and COCHRAN, V.G. - Two or more randon samples of measurement data. Analysis of variance in: Statistical Univer syte Press, 1956. cap 10, p. 246-247.

21. SWAROOP, A., GRAB, M. and OHSAKA, A. - Henorragic Necrotizing and Edema-Forming Effects of Snake Venouns. Chapter 14. Washington MaC Graw Hill, 1975, 503 p.

22. VONDRUSKA, J.F. and GRECO, R.A. - Certaim hematologic and blood chemical values em charles river C D Albino Rats. Buzz, Am Soc. Vet. Clin. Pathol, 2:3-7, 1973.

23. WALKER, A.I.I., STEVENSON, D.E., ROBINSON, J.; THORPE, E. and 
ROBERTS, M. - The toxicity and pharmacodynamics of dieldrim (Hedd), Toxical. appe. Pharmacol, 15:345-373, 1969.

24. WORDEN, A., NOEL, P.R.B., MAWDESLEY, T.L.E., PALMER, A.K. and FLETCHER, M.A. - Teading studies on lenacil in the rat and dog. Toxicol. appe. Pharmaçol. 27:215-224, 1974.

Recebido em abril, 1987; aceito em dezembro, 1987. 
